\title{
COMPATIBILIDAD ENTRE LA CONSERVACIÓN DE LA DIVERSIDAD BIOLÓGICA Y LA PRODUCCIÓN EN UNA PLANTACIÓN FORESTAL EN LA PATAGONIA NOROCCIDENTAL ARGENTINA
}

\author{
Dezzotti, Alejandro5 ; Sbrancia, Renato; Mortoro, Ariel y Attis Beltrán, Hernán
}

\section{RESUMEN}

La diversidad biológica y la productividad de un ecosistema representan indicadores clave de integridad ambiental que son afectados significativamente por la introducción de especies. Las plantaciones forestales con árboles exóticos proveen bienes y servicios imprescindibles para la humanidad, aunque disminuyen la riqueza de plantas y animales cuando conforman monocultivos coetáneos de alta densidad.

Este estudio evaluó la compatibilidad ambiental y económica de una plantación de Pinus ponderosa (Pinaceae), localizada en el campo forestal Litrán del noroeste de la Patagonia argentina $\left(38^{\circ} 54^{\prime} \mathrm{S}\right.$ y $71^{\circ} 01^{\prime} \mathrm{O}, 1.400 \mathrm{msnm}$., $\left.1.310 \mathrm{ha}\right)$. El análisis involucró la medición de parámetros físicos y biológicos y la producción de madera en comunidades con una variada estructura poblacional.

En el área de estudio, el invierno es frío y húmedo y el verano cálido y seco. La litología corresponde a basaltos y andesitas cubiertas por tefra Holocénica, que proporcionan el material parental volcánico de los Andisoles predominantes. La vegetación corresponde a la transición entre la estepa patagónica de pastos y arbustos y el bosque subantártico.

Los pinos se plantaron en la estepa entre 1991 y 1995 y actualmente conformaron rodales abiertos (RA, $<15 \mathrm{~m}^{2} /$ ha área basal), intermedios $\left(\mathrm{RI}, 15-30 \mathrm{~m}^{2} / \mathrm{ha}\right)$ y cerrados $(\mathrm{RC},>30$ $\mathrm{m}^{2} / \mathrm{ha}$ ). En cada categoría se estimó la producción de madera, con el modelo regional de rendimiento para $P$. ponderosa "Piltriquitrón" con un turno de corta de 36 años. En cada rodal y en el pastizal circundante de referencia se estimaron la diversidad de plantas y la cantidad de suelo desnudo a través de datos de incidencia presencia / ausencia.

Los rodales presentaron la misma calidad de sitio y una cobertura aérea de $29 ; 63$ y $91 \%$ en RA, RI y RC, respectivamente. En RA, RI y RC, la producción total de madera se estimó en 334 , 426 y $456 \mathrm{~m}^{3} / \mathrm{ha}$, respectivamente, mientras que la productividad total en 9,$3 ; 11,8$ y 12,7 $\mathrm{m}^{3} /$ ha/año, respectivamente. En $R A, R I$ y $R C$, la riqueza de plantas fue 30,16 y 16 especies, respectivamente, mientras que el índice de diversidad fue 5,$2 ; 1,7$ y 1,3 , respectivamente. La riqueza y diversidad de plantas del pastizal fue 45 y 6,4 , respectivamente. En RA, la producción de madera fue $32 \%$ menor, la riqueza de especies fue el doble, la diversidad fue hasta 3,5 veces mayor y la proporción de suelo desnudo fue tres veces menor a la de RI y RC.

Estudios previos en sistemas productivos equivalentes encontraron una diversidad menor a la del pastizal, que aumentó en rodales estructuralmente más complejos. La correlación negativa que existió entre la productividad y la diversidad se debe tener en cuenta para implementar escenarios de sustentabilidad en estos hábitats antropogénicos. La compatibilidad entre la conservación de especies silvestres y la producción de madera requiere esencialmente mantener rodales abiertos a lo largo del ciclo de corta. Aunque tales proyectos forestales puedan presentar una productividad de madera menor, podrán tener un valor ecosistémico y estético mayor que aumentará la posibilidad de sostenerlos económicamente por parte de la sociedad.

Palabras clave: Pinus ponderosa, índice de diversidad de Simpson, producción de madera.

5 Ph. D. Ecología Forestal, Profesor Adjunto. Universidad Nacional del Comahue, San Martín de los Andes, Argentina.dezzotti@infovia.com.ar. 


\section{SUMMARY}

Biodiversity and productivity are ecosystem integrity key indicators that are significantly affected by species introduction. Forest plantations based on exotic trees provide goods and services essential to humanity, although they diminish plant and animal richness when constitute even-aged monocultures of large density.

In this study the environmental and economic sustainability of a Pinus ponderosa (Pinaceae) plantation, located in Litrán within northwestern Patagonia of Argentina (38 $54^{\prime} \mathrm{S}$ and $71^{\circ} 01^{\prime} \mathrm{W}, 1,400 \mathrm{masl}, 1,310 \mathrm{ha}$ ), was evaluated. The analysis involved physical and biological variables and wood production, measured in communities with contrasting population structures.

In the study area, winter is cold and wet and summer is warm and dry. Lithology comprised basalts and andesites covered by Holocene tephra, which provide the volcanic parent material of the predominant Andisols. Vegetation corresponded to the transition between the Patagonian semirarid steppe of grasses and shrubs and the subantarctic temperate forest.

Pines were planted between 1991 and 1995 in the steppe, and at present, they constituted open (RA, $<15 \mathrm{~m}^{2} /$ ha basal area), intermediate (RI, $15-30 \mathrm{~m}^{2} /$ ha) and closed stands $\left(\mathrm{RC},>30 \mathrm{~m}^{2} / \mathrm{ha}\right)$. In these structural classes, timber production was estimated with the regional production model for $P$. ponderosa "Piltriquitrón" with a rotation length of 36 years. In each stand and in the surrounding grassland as the reference community, plant diversity and bare soil were estimated through incidence presence / absence data.

Stands exhibited an equivalent site quality and canopy cover was $29 ; 63$ and $91 \%$ for RA, $\mathrm{RI}$ and RC, respectively. In RA, RI and RC, final timber production was 334,426 and $456 \mathrm{~m}^{3} / \mathrm{ha}$, respectively, and productivity was $9.3,11.8$ and $12.7 \mathrm{~m}^{3} / \mathrm{ha} / \mathrm{yr}$., respectively. In RA, RI and RC, plant richness was 30, 16 and 16 species, respectively, and diversity index was 5.2, 1.7 and 1.3, respectively. Plant richness and diversity of grassland were 45 species and 6.4, respectively. In RA timber production was $32 \%$ lower, richness twice, diversity up to 3.5 times higher and bare soil proportion three times lesser in comparison to $\mathrm{RI}$ and $\mathrm{RC}$.

Previous studies in analogous production systems found a lower diversity compared to grassland, but it increased in structurally more complex stands. The negative correlation between timber production and biological diversity should be considered for implementing sustainability scenarios within these anthropogenic habitats. The compatibility between wildlife preservation and timber production basically requires keeping open stands along the entire production cycle. Although such projects would represent a lower economic income from timber, they would have a larger ecosystem and aesthetic value that will increase the possibility of supporting them by society.

Key words: Pinus ponderosa, Simpson diversity index, timber production. 


\section{INTRODUCCIÓN}

En los últimos 50 años, el hombre modificó los ecosistemas de la manera más rápida, intensa y extendida que en ningún otro momento de la historia de la humanidad, afectando en forma dramática a los bosques naturales. En la actualidad, la pérdida anual de estos ecosistemas se estima en 13 millones de hectáreas, principalmente debido a su conversión a tierras agrícolas, ganaderas y urbanas (Lindquist et al., 2012; FAO, 2014). América del Sur exhibe la mayor tasa neta de pérdida de bosques, que alcanza 3,3 millones de hectáreas por año y que equivale a aproximadamente 22,6\% de la pérdida forestal global (Hansen et al., 2010; Lindquist et al., 2012).

A lo largo del siglo XX, la Argentina perdió $70 \%$ de sus bosques naturales (SAyDS, 2007). Al mismo tiempo que se produjo este proceso de degradación y destrucción, la demanda de bienes y servicios provenientes de estos ecosistemas forestales continuó en aumento. Por ejemplo, el consumo de madera se triplicó y el de papel se sextuplicó desde 1950 , el $\mathrm{CO}_{2}$ atmosférico alcanzó en la actualidad el nivel máximo y en muchas regiones del mundo incluida la Patagonia, la tasa de erosión superó la de formación de suelo (FAO, 2014). La pérdida de la cubierta arbórea y el cambio de uso puede afectar al clima al alterar el balance de carbono y la reflectancia de la superficie del suelo (Feddema, et al., 2005; Pan et al., 2011).

El desajuste entre el aumento de la demanda y la disminución de la oferta de recursos forestales explica en parte la creación de plantaciones forestales, estas constituyen "bosques que se establecen por siembra o plantación de especies nativas o exóticas, a través de programas de forestación y reforestación" (FAO, 2014). Las plantaciones son típicamente monocultivos coetáneos, silvicultural y geográficamente intensivos, que producen madera a mayor velocidad que los bosques naturales (Kanowski, 1997). Las plantaciones de coníferas de la Patagonia se localizan sobre la faldeos andinos semiáridos de las provincias de Neuquén, Río Negro y Chubut y ocupan alrededor de 100.000 ha (MAGyP, 2014). En esta región, la actividad forestal comercial comenzó en la década del 1970 en Neuquén y las plantaciones actuales son establecidas en áreas de secano que no presentan bosques naturales. Neuquén posee el $60 \%$ de la superficie de estas plantaciones, Pinus ponderosa (Pinaceae) se extiende en el $80 \%$ de la superficie total, el resto corresponde a Pinus contorta y Pseudotsuga menziesii (Pinaceae) (MAGyP, 2014).

Las plantaciones se asocian al control de la desertificación, la protección del suelo (La Manna et al., 2013), la restauración de áreas degradadas (Frugoni et al., 2016) y el secuestro de carbono (Laclau, 2003; Nosetto et al., 2006). También contribuyen a la conservación de la biodiversidad (Lindenmayer et al., 2006; Brockerhoff et al., 2008; Bremer y Farley, 2010). Las plantaciones exhiben un conjunto de riesgos ambientales asociados a la invasión de Pinus hacia los ambientes naturales circundantes (Price et al., 1998; Sarasola et al., 2006; Dezzotti et al., 2009), al mayor consumo de agua (Le Maitre et al., 1999; Gyenge et al., 2011) y al aumento de los incendios forestales (Loguercio et al., 2011). En la Argentina, el reemplazo de bosques naturales y pastizales por plantaciones de $P$. ponderosa provocó una biodiversidad menor de plantas, insectos, aves y mamíferos nativos en plantaciones densas, con una pérdida de especies raras y especialistas, en comparación con los valores de los sistemas originales y de las plantaciones ralas (Corley et al., 2006; Lantschner et al., 2008, 2011; Paritsis y Aisen, 2008).

La producción, el crecimiento y la forma del fuste de los árboles de una especie en un rodal dependen de la calidad de sitio y la densidad (Daniel et al., 1979). En las plantaciones de la Patagonia de la Argentina, el factor clave de manejo es la densidad y existen escasas experiencias de modificación del sitio. El crecimiento diametral del tronco aumenta a medida que disminuye la densidad, y en consecuencia, para una determinada edad los rodales que se desarrollan en forma más espaciada tendrán un diámetro promedio mayor que aquellos que presentan árboles menos espaciados. Teniendo en cuenta que el crecimiento en diámetro es muy sensible a la densidad, el bosque se debe mantener bastante abierto si se desea producir árboles más gruesos. El manejo de la densidad se efectúa mediante raleos, que consisten en extraer determinados árboles para que los remanentes dispongan de mayor cantidad de recursos y el crecimiento se concentre en los mejores individuos. Esta técnica incrementa la cantidad y calidad de la materia prima comercial y el valor individual, y disminuye el turno de corta (Loguercio et al., 2011). 


\section{OBJETIVOS}

Los objetivos de este proyecto fueron:

-Evaluar el valor de indicadores de conservación de una plantación, implementada con tres niveles de intensidad de raleo, y de la estepa herbáceo-arbustiva como unidad de paisaje de referencia.

-Evaluar la producción de madera de la plantación.

-Evaluar la relación entre los indicadores de conservación y producción.

-Proponer prescripciones técnicas de conservación de la diversidad a escala de rodal.

\section{MATERIAL Y MÉTODO}

El área de estudio comprendió la estación forestal Litrán $\left(38^{\circ} 54^{\prime} \mathrm{S}\right.$ y $71^{\circ} 01^{\prime} \mathrm{O}, 1.400$ msnm, 1.310 ha). El clima es húmedo y ventoso, con inviernos fríos y veranos cálidos (AIC, 2012) (Figura $\mathrm{N}^{\circ} 1$ ). La clasificación climática de Köppen-Geiger califica la zona como Csb (Peel et al., 2007).
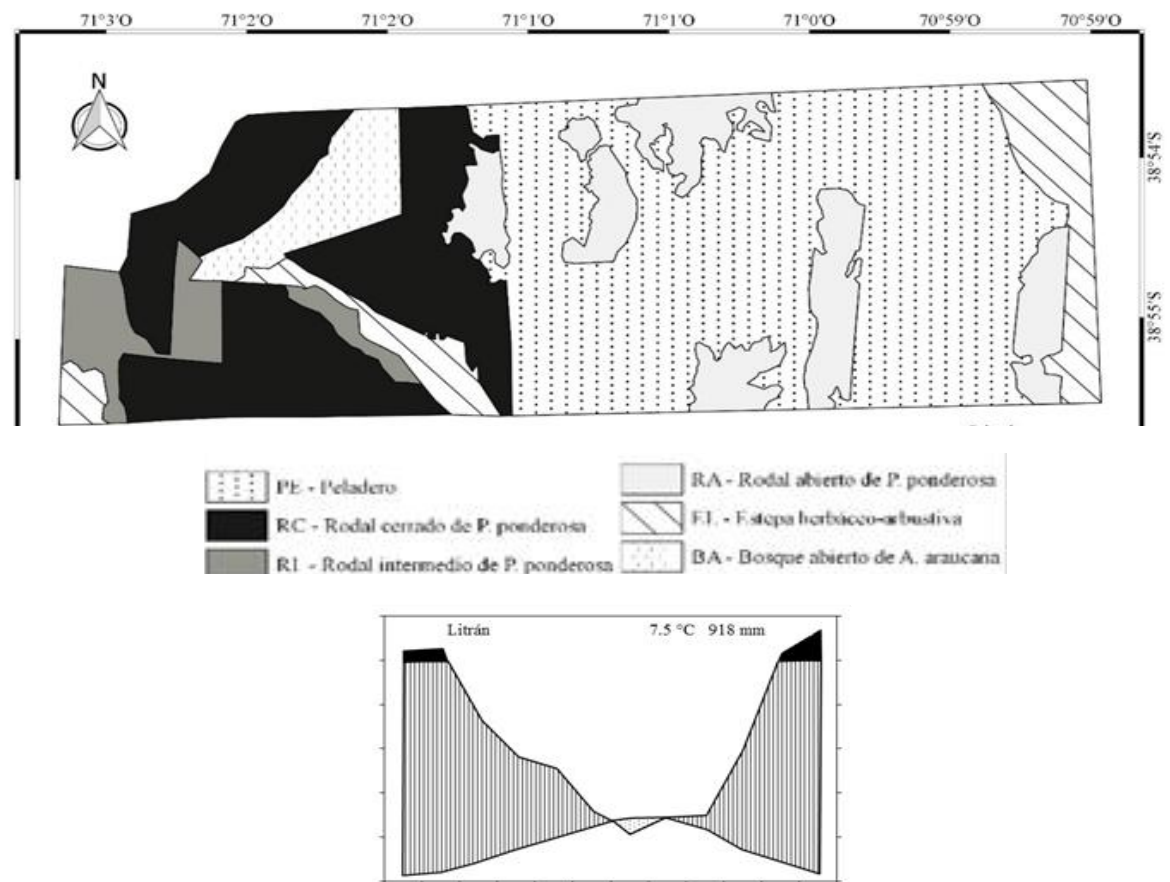

Figura $\mathrm{N}^{\circ} 1$

UNIDADES DE PAISAJE Y DIAGRAMA CLIMÁTICO DEL CAMPO FORESTAL LITRÁN LOCALIZADO EN EL NOROESTE DE LA PROVINCIA DE NEUQUÉN 
La geología de base corresponde a basaltos, andesitas, brechas, aglomerados volcánicos y depósitos glaciales no estratificados. Estos elementos están cubiertos por una capa de tefra Holocénica, suministrada por los volcanes activos de la cordillera de Los Andes, que conforma el material parental de los suelos. La geomorfología consiste en montañas con signos de glaciación y erosión como lagos, circos, valles colgados y bloques erráticos. Los suelos dominantes corresponden a Andisoles Udivitrands y Endoaquands alofánicos (Frugoni et al., 2016).

El área de estudio pertenece a la transición entre la ecorregión Patagónica Semiárida y la Subantártica Húmeda (Cabrera, 1971). El tipo de vegetación más extensa es la estepa que ocupa las zonas más bajas sobre suelos zonales. Los bosques altos puros y mixtos dominados por Nothofagus (Nothofagaceae) están presentes hacia el oeste en laderas de elevación intermedia y de diferente exposición, y los bosques abiertos de Araucaria araucana (Araucariaceae) y Nothofagus antarctica ocupan zonas orientales rodeados por la estepa. Las praderas xerófilas (pedreros) se desarrollan en afloramientos rocosos basálticos y andesíticos y las higrófilas (mallines) en suelos intrazonales de los sectores más bajos y mal drenados. El área es parte de una ruta histórica de la ganadería nómade de subsistencia, de cabras y ovejas, que migra desde los valles bajos en invierno a las elevaciones más altas en verano. La vegetación tiende a presentar un intenso sobrepastoreo y quema debido a esta práctica tradicional (Frugoni et al., 2016).

Los árboles de Litrán fueron plantados sobre suelos volcánicos profundos y bien drenados de la estepa entre 1991 y 1995, la plantación está protegida por un alambrado perimetral contra los grandes herbívoros domésticos y silvestres y se implementó un sistema de control de incendios.

Las unidades de paisaje naturales y antropogénicas del campo forestal Litrán fueron identificados en una imagen de satélite Aster (resolución 15 m, Gauss Krüger coordenadas banda 1, elipsoide WGS 1984), utilizando la fisonomía, la composición y estructura de la vegetación y uso del suelo como criterios orientadores, y la cartografía se verificó en el campo.

Los rodales de $P$. ponderosa que ocuparon las unidades de paisaje antropogénicas se clasificaron de acuerdo a la cobertura de árboles en abierto (RA, área basal $<15 \mathrm{~m}^{2} / \mathrm{ha}$ ), intermedio (RI, $15-30 \mathrm{~m}^{2} / \mathrm{ha}$ ) y cerrado (RC, > $30 \mathrm{~m}^{2} / \mathrm{ha}$ ) basado en un inventario forestal previo (CFI, 2009). En cada categoría estructural, se instaló al azar una parcela de $400 \mathrm{~m}^{2}$ y se midió el diámetro a la altura del pecho $\left(d_{b}, 1,3 \mathrm{~m}\right.$ del suelo). En cada parcela se seleccionaron cuatro árboles con el tronco más grueso y uno con el más delgado y se les midió la longitud de los cinco entrenudos $\left(I_{5}\right)$ por encima de $1,3 \mathrm{~m}$ del suelo (cinta métrica).

La calidad de sitio de cada rodal, que representa la máxima productividad potencial de una especie o tipo forestal en un sitio determinado (Daniel et al., 1979), se determinó a partir del índice de sitio (IS), que se expresa como la altura promedio de los árboles dominantes del rodal a una edad de referencia. Para $P$. ponderosa se utilizó 20 años a la altura del pecho $\left(I S_{20}\right)$ (Andenmatten y Letorneau, 2003) de acuerdo a:

$$
I S_{20}=4,37+14,86 \frac{I_{5}}{5}
$$

En cada categoría se estimó la producción de madera con el modelo regional de rendimiento para $P$. ponderosa "Piltriquitrón" con un turno de corta de 36 años. Este simulador de crecimiento y rendimiento de madera a escala de rodal se basa en el índice de sitio, el espaciamiento $(d, \mathrm{~m})$, el factor de altura de los árboles $(F H, \mathrm{~m} / \mathrm{m})$, el diámetro cuadrático medio $\left(D_{g}\right.$, $\mathrm{cm} / \mathrm{ind})$, la densidad relativa $\left(D_{r}, \%\right)$ y el área basal $\left(A B, \mathrm{~m}^{2} / \mathrm{ha}\right)$ (Andenmatten et al., 2007), de acuerdo a:

$$
d=\sqrt{\frac{10,000}{n}}
$$




$$
\begin{aligned}
& F H=\frac{\text { Altura dominante }}{d} \\
& D_{g}=\sqrt{\frac{4 A B}{n-\pi}} \\
& D_{r}=\frac{A B}{\sqrt{D g}}
\end{aligned}
$$

En cada una de las categorías estructurales de los rodales de $P$. ponderosa (RA, RI y RC) y en la estepa herbácea-arbustiva circundante (EL) se instalaron en forma sistemática y equidistante 10 sitios de muestreo ubicados dentro de los límites del campo forestal. En cada sitio de muestreo se registró la posición geográfica (GPS), altitud (altímetro), pendiente (clinómetro y cinta métrica) y exposición (brújula).

El ambiente luminoso bajo el dosel de árboles se caracterizó midiendo la radiación fotosintéticamente activa y se expresó como la intensidad relativa de luz con respecto a un área adyacente completamente abierta (receptor de luz LI-250 y el sensor cuántico LI-190SA, LI COR, Inc.).

La densidad aparente del suelo de cada unidad de paisaje se estimó mediante el método del cilindro colectando al azar durante la estación seca, tres muestras de suelo del horizonte A con cilindro de metal compuesto de tres anillos móviles con un volumen conocido y, a continuación, se pesaron en el laboratorio (balanza de precisión) (SAMLA, 1996).

El contenido de humedad del suelo se estimó mediante la extracción de tres muestras de suelo que se almacenaron herméticamente y pesaron en el laboratorio, luego se secaron durante $24 \mathrm{~h}$ a $90^{\circ} \mathrm{C}$ y se pesaron nuevamente (balanza de precisión, horno).

Desde el centro de cada sitio de muestreo, se establecieron cuatro transectos de $10 \mathrm{~m}$ a lo largo de las direcciones cardinales N, S, E y O. En cada transecto, se localizó cada $1 \mathrm{~m}$ un punto de muestreo para determinar la presencia de suelo desnudo y plantas vasculares, incluyendo plantas y árboles jóvenes, utilizando el método del punto de intersección (Kent, 2011). Las plantas se clasificaron de acuerdo a la especie, el ciclo de vida (perenne, anual y bianual) y el origen (nativa e introducida) según IBD (2015). La frecuencia basada en los datos de incidencia (presencia / ausencia) es una variable adecuada para medir la importancia de plantas que no están adaptados a mediciones apropiadas de densidad, biomasa, uso de energía o cobertura (Jost et al., 2010). En el caso de las plantas esta aproximación es adecuada teniendo en cuenta las limitaciones que surgen cuando se quiere establecer con claridad cuál es el límite físico de cada individuo para determinar la abundancia de las especies (Magurran y McGill, 2011). La diversidad taxonómica se estimó a través del índice de Simpson (1949), de acuerdo a:

$$
\begin{aligned}
& D=\frac{1}{\sum_{i}^{5} p_{i}^{2}} \\
& E=\frac{D}{s}
\end{aligned}
$$

Donde: $D$ : diversidad taxonómica, $S$ : riqueza de especies, $E$ : equitatividad y $P_{i}$ : proporción de individuos de la especie $i$. La similitud entre unidades de paisaje se determinó a través del coeficiente de Sørensen (1948) de acuerdo a:

$$
C_{s}=\frac{2 s_{12}}{s_{1}+s_{2}}
$$

Donde $C_{S}$ : coeficiente de similitud, $S_{1}$ y $S_{2}$ : cantidad total de especies en las comunidades 1 y 2 y $S_{12}$ cantidad de especies comunes. La dominancia de las especies en cada unidad de paisaje se estimó a través de coeficiente de McNaughton y Wolf (1970) de acuerdo a:

$$
C_{M C N}=\frac{p_{1}+p_{2}}{\sum_{i=1}^{S} p_{i}^{i}} \times 100
$$


Donde $C_{M C N}$ : valor de dominancia (\%), $P_{1}$ y $P_{2}$ : abundancia de las dos especies más abundantes (\%) y $P_{i}$ : abundancia de la especie $i$. La cantidad de especies raras se determinó a partir del índice $R_{\text {logskew }}$ (McGill, 2003), de acuerdo a:

$R_{\text {Log shew }}=\frac{\sum_{i=1}^{s} \frac{\left(\log n_{i}-\mu\right)^{3}}{s}}{\left(\sum_{i=1}^{s} \frac{\left(\log n_{i}-\mu\right)}{s}\right)^{\frac{3}{2}}} \frac{s}{s-2 \sqrt{\frac{5-1}{s}}}$

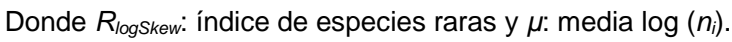

\section{RESULTADOS}

Las plantaciones de pino con densidad y cobertura baja (RA), intermedia (RI) y alta (RC) exhibieron diferencias de la densidad, el área basal, el diámetro y la altura media de los árboles adultos. Estas variables exhibieron los valores menores en RA, mientras que presentaron los mayores en RC. La densidad varió entre 350 y 775 ind/ha, el área basal entre 11,1 y $45,3 \mathrm{~m}^{2} / \mathrm{ha}$ y la altura media entre 7,5 y 13,2 m/ind. Aunque el índice de sitio difirió entre las categorías de rodales y fue 12,$6 ; 13,2$ y 14,6 m para $\mathrm{RA}, \mathrm{RI}$ y $\mathrm{RC}$, respectivamente, estos valores pertenecen a la misma clase de calidad (Cuadro $\mathrm{N}^{\circ} 1$ ).

\section{Cuadro $\mathrm{N}^{\circ} 1$}

PARÁMETROS ESTRUCTURALES Y PRODUCTIVOS DE LOS RODALES DE LITRÁN.

\begin{tabular}{|l|ccc|ccc|ccc|}
\hline \multirow{2}{*}{\multicolumn{1}{|c|}{ VARIABLE }} & \multicolumn{3}{c|}{ Rodal Abierto (RA) } & \multicolumn{3}{c|}{ Rodal Intermedio (RI) } & \multicolumn{3}{c|}{ Rodal Cerrado (RC) } \\
\cline { 2 - 10 } & INI & POS & FIN & INI & POS & FIN & INI & POS & FIN \\
\hline Edad (años) & 10 & 22 & 36 & 18 & 30 & 36 & 20 & 22 & 36 \\
Densidad (ind/ha) & 350 & 150 & 150 & 400 & 275 & 275 & 775 & 525 & 375 \\
Área basal (m²/ha) & 11,1 & 17,5 & 31,7 & 24,6 & 31,7 & 39,0 & 45,3 & 38,0 & 47,2 \\
Densidad relativa (\%) & 2,5 & 2,8 & 4,4 & 4,7 & 5,1 & 6,0 & 8,7 & 6,9 & 7,5 \\
Volumen (m ${ }^{3} /$ ha) & 41,9 & 107,4 & 273,8 & 135,3 & 244,8 & 340,5 & 248,2 & 225,6 & 394,5 \\
Diámetro cuadrático (cm/ind) & 20,1 & 38,5 & 51,9 & 28,0 & 38,3 & 42,5 & 27,3 & 30,3 & 40,0 \\
Altura media (m/ind) & 7,5 & 14,3 & 20,7 & 12,3 & 18,2 & 20,7 & 13,2 & 14,2 & 20,4 \\
Distanciamiento (m) & 5,3 & 8,2 & 8,2 & 5,0 & 6,0 & 6,0 & 3,6 & 4,4 & 5,2 \\
Factor de altura (m/m) & 1,4 & 1,8 & 2,5 & 2,5 & 3,0 & 3,4 & 3,7 & 3,2 & 4,0 \\
índice de sitio (m) & 12,6 & 12,6 & 12,6 & 13,2 & 13,3 & 13,3 & 14,6 & 14,6 & 14,6 \\
\hline
\end{tabular}

Se indican los valores iniciales (INI), post-raleo (POS) y de corta final (FIN)

Los árboles en RA se localizaron en forma balanceada en las clases de tamaño 1 (10 $19,9 \mathrm{~cm}$ DAP) y 2 (20 - $29 \mathrm{~cm}$ DAP), mientras que los de RI y RC se localizaron principalmente en la clase $2\left(\mathrm{RI}, 81,3 \%\right.$ y $\mathrm{RC}, 77,4 \%$ del total) (Figura $\left.\mathrm{N}^{\circ} 2\right)$.

La radiación en el piso del bosque fue 42,1\% $(E E=3,9)$ para $\mathrm{RA}, 16,3 \%(\mathrm{EE}=7,3)$ para $\mathrm{RI}$ y $2,0 \%(\mathrm{EE}=0,5)$ para RC; estos valores difirieron estadísticamente entre sí (prueba LSD, ANOVA $_{2,29} \mathrm{~F}=17,9 ; \mathrm{P}<0,0001 ; \mathrm{n}=10$ ).

La densidad aparente del suelo en $E L, R A, R I$ y $R C$ se estimó en 0,78 ( $E E=0,04$, mínimo $0,72 \mathrm{~g} / \mathrm{cm}^{3}$ y máximo $\left.0,84 \mathrm{~g} / \mathrm{cm}^{3}\right), 0,77(\mathrm{EE}=0,05 ; 0,68$ y 0,85$), 0,71(\mathrm{EE}=0,13 ; 0,46$ y $0,89)$ y $0,73 \mathrm{~g} / \mathrm{cm}^{3}(E E=0,07 ; 0,58$ y 0,82$)$, respectivamente; estos valores no difirieron estadísticamente entre sí $\left(\mathrm{ANOVA}_{3,11}, \mathrm{~F}=0,14 ; \mathrm{P}=0,936 ; \mathrm{n}=3\right.$ ).

El contenido de humedad del suelo de EL, RA, RI y RC fue 18,3 (EE = 0,7; mínimo 17,1\% 
y máximo $19,3 \%), 19,9$ ( $E E=0,4 ; 19,2$ y 20,7), 9,9 ( $E E=1,4 ; 7,3$ y 11,8$)$ y $9,6 \%(E E=0,9 ; 8,1$ y 11,2 ); estos valores difirieron estadísticamente entre sí (prueba LSD, $\mathrm{ANOVA}_{3,11} \mathrm{~F}=36,4 ; \mathrm{P}=$ 0,$0001 ; n=3$ ) (Figura No 3 ).

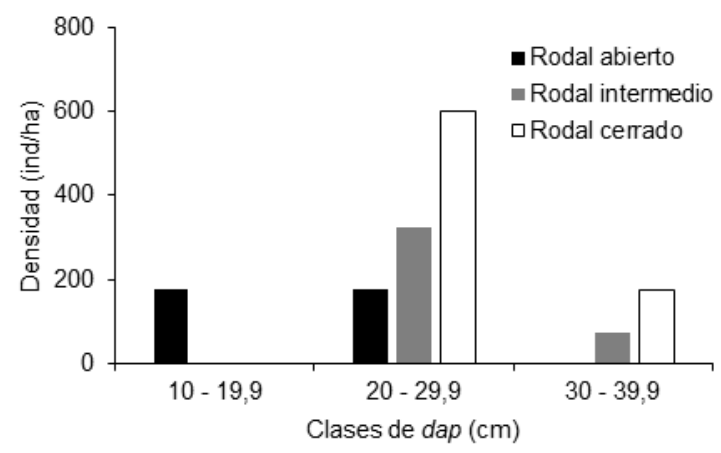

Figura $\mathrm{N}^{\circ} 2$

DISTRIBUCIÓN DE LAS CLASES DE DIÁMETRO (DAP) DE LOS RODALES DE LITRÁN
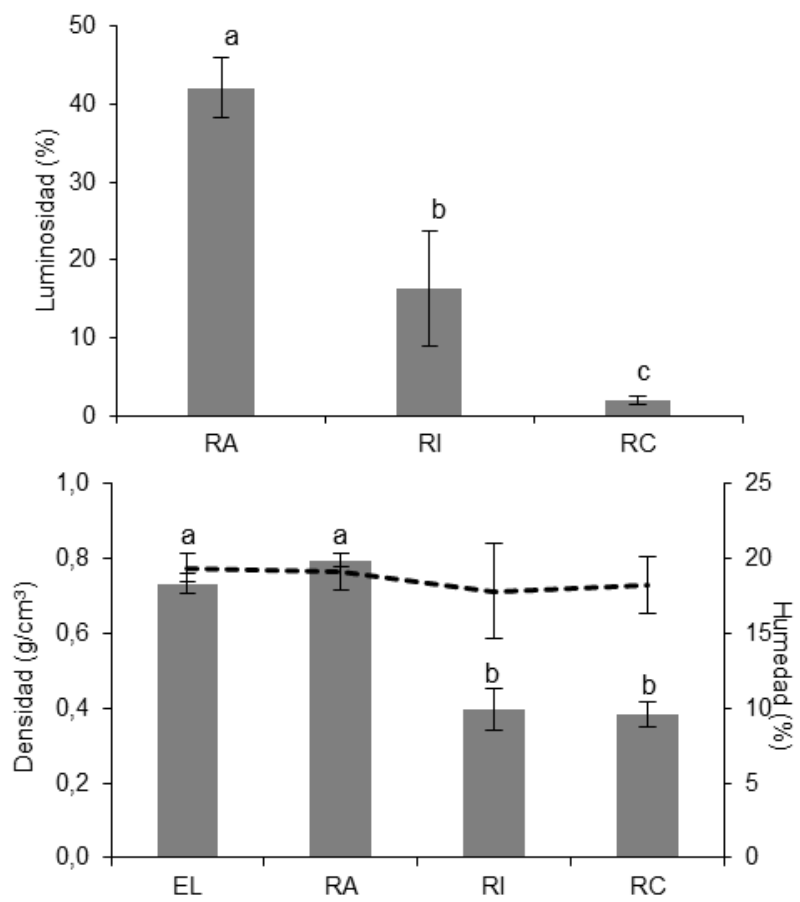

Las líneas verticales indican el error estándar de la media y las letras diferentes las diferencias significativas de las medias (prueba LSD, $p<0,05$ )

Figura $N^{\circ} 3$

LUMINOSIDAD (ARRIBA) Y DENSIDAD (LÍNEA PUNTEADA) Y HUMEDAD DEL SUELO (BARRA) (ABAJO) EN LA ESTEPA-HERBÁCEO-ARBUSTIVA (EL) Y LOS RODALES ABIERTO (RA), INTERMEDIO (RI) Y CERRADO (RC) 
La cobertura arbórea en los rodales varió entre $28,6 \%$ en RA y $90,9 \%$ en RC; estos valores difirieron estadísticamente entre sí (prueba LSD, ANOVA $_{3,39} \mathrm{~F}=100,6 ; \mathrm{P}<0,0001 ; \mathrm{n}=10$ ).

La cantidad de suelo desnudo fue $28,0 \%(E E=2,9)$ para $E L, 28,2 \%(E E=3,7)$ para $R A$, $83,6 \%(E E=3,8)$ para $\mathrm{RI}$ y $89,3 \%(E E=3,6)$ para $R C$; estos valores difirieron estadísticamente entre sí (prueba LSD, ANOVA ${ }_{3,39} \mathrm{~F}=92,0 ; \mathrm{P}<0,0001 ; \mathrm{n}=10$ ) (Figura $\mathrm{N}^{\circ} 4$ ).

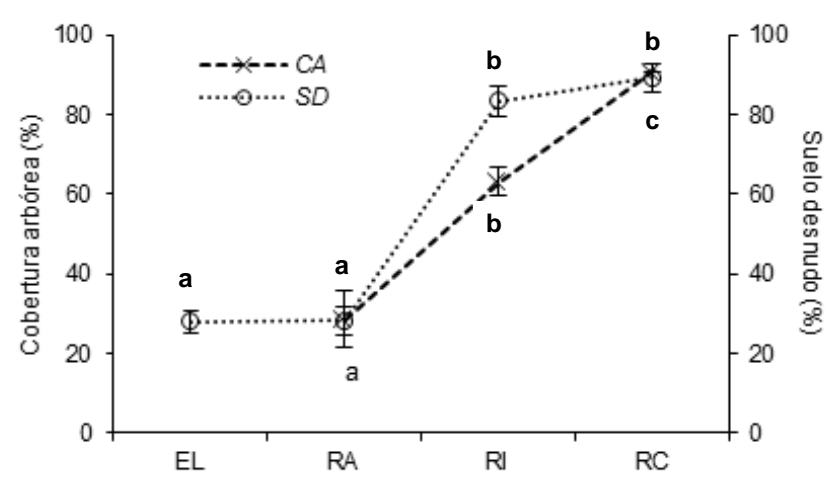

Las líneas verticales indican el error estándar de la media y las letras diferentes las diferencias significativas de las medias entre unidades de paisaje (prueba LSD, $p<0,05$ )

Figura $N^{\circ} 4$

COBERTURA ARBÓREA (CA) Y CANTIDAD DE SUELO DESNUDO (SD) EN LA ESTEPA-HERBÁCEOARBUSTIVA (EL) Y LOS RODALES ABIERTO (RA), INTERMEDIO (RI) Y CERRADO (RC)

La riqueza de especies de plantas vasculares varió entre 3,6 (RC, $E E=0,8)$ y 14,8 (EL, $\mathrm{EE}=1,0$ ) y estos valores difirieron estadísticamente entre sí (prueba LSD, ANOVA $\mathrm{A}_{3,39} \mathrm{~F}=42,9, \mathrm{P}<$ $0,0001 ; n=10)$, mientras que la diversidad varió entre $1,3(R C, E E=0,1)$ y $6,1(E L, E E=1,0)$ y estos valores difirieron estadísticamente entre sí (prueba LSD, ANOVA $_{3,39} F=21,6 ; P<0,0001, n=$ 10).

La cantidad de especies nativas en las cuatro unidades de paisaje varió entre 9,7\% (RC, $E E=2,9)$ y $82,1 \%(E L, E E=0,9)$ y estos valores difirieron estadísticamente entre sí (prueba LSD, ANOVA $_{3,39} F=186,1, P<0,0001, n=10$ ) (Figura $N^{\circ} 5$ ).

El cociente cantidad de especies y familias (S : F) varió entre $1,1(R C)$ y $2,0(E L)$ y los valores difirieron estadísticamente entre sí (prueba LSD, ANOVA $A_{3,39} F=15,8, P<0,0001, n=10$ ).

La dominancia de especies de plantas $\left(C_{M c N}\right)$ para EL, RA, RI y RC fue 18,$4 ; 28,3 ; 44,4$ y $46,1 \%$; respectivamente.

La cantidad de especies raras ( $R_{\text {logskew }}$ ) varió de 0,53 en EL y 2,29 en RC (Figura N 6).

Los valores mayores de similitud $\left(C_{s}\right)$ entre las unidades de paisaje se presentaron entre EL y RA $(0,59)$ y entre RI y RC $(0,44)$, mientras que los menores entre $E L$ y $R I(0,19)$ y $E L$ y $R C$ $(0,22)$ (Figura $\mathrm{N}^{\circ} 7$ ). 

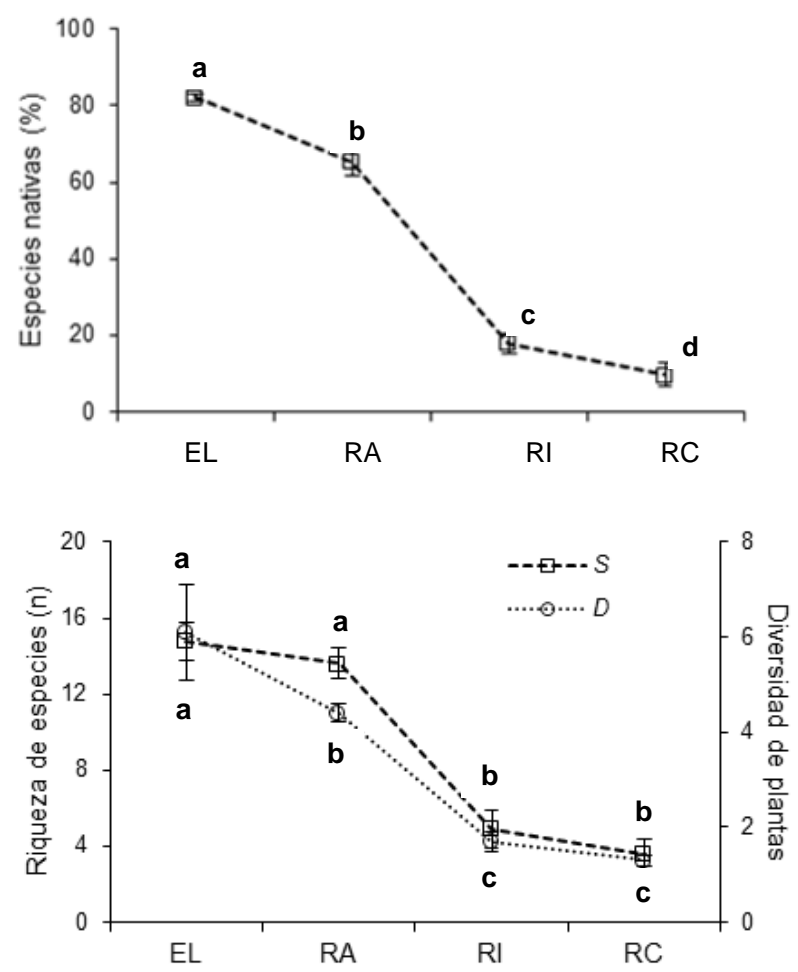

Las líneas verticales indican el error estándar de la media y las letras diferentes las diferencias significativas de las medias entre unidades de paisaje (prueba LSD, $\mathrm{p}<0,05$ )

\section{Figura $\mathrm{N}^{\circ} 5$}

\section{CANTIDAD DE ESPECIES NATIVAS (ARRIBA) Y RIQUEZA (S) Y DIVERSIDAD DE PLANTAS (D) (ABAJO) EN LA ESTEPA-HERBÁCEO-ARBUSTIVA (EL) Y LOS RODALES ABIERTO (RA), INTERMEDIO (RI) Y CERRADO (RC)}

El simulador de rendimiento a escala de rodal para $P$. ponderosa estimó una producción total de madera a los 36 años, de $333,9 \mathrm{~m}^{3} /$ ha para RA $\left(294,0 \mathrm{~m}^{3} /\right.$ ha de madera gruesa, rollizos > $\left.20 \mathrm{~cm} \mathrm{~d}_{b}\right), 426,2 \mathrm{~m}^{3} /$ ha para RI $\left(373,0 \mathrm{~m}^{3} / \mathrm{ha}\right)$ y $455,6 \mathrm{~m}^{3} / \mathrm{ha}$ para RC $\left(371,0 \mathrm{~m}^{3} / \mathrm{ha}\right)$.

La productividad total la estimó en $9,3 \mathrm{~m}^{3} /$ ha/año $\left(8,2 \mathrm{~m}^{3} /\right.$ ha/año de madera gruesa) para RA; $11,8 \mathrm{~m}^{3} /$ ha/año $\left(10,4 \mathrm{~m}^{3} /\right.$ ha/año) para $R \mathrm{Rl}$ y $12,7 \mathrm{~m}^{3} /$ ha/año $\left(10,3 \mathrm{~m}^{3} /\right.$ ha/año).

La producción total de madera al final del ciclo productivo aumentó $27,6 \%$ en $\mathrm{RI}$ en comparación con RA y $36,5 \%$ en RC en comparación con RA, mientras que solo $6,9 \%$ en RC en comparación con RI.

Al tiempo que aumentó la cantidad de madera a medida que los rodales presentan una mayor densidad y cobertura, la diversidad de plantas disminuyó (Figura $\mathrm{N}^{\circ} 8$ ). 

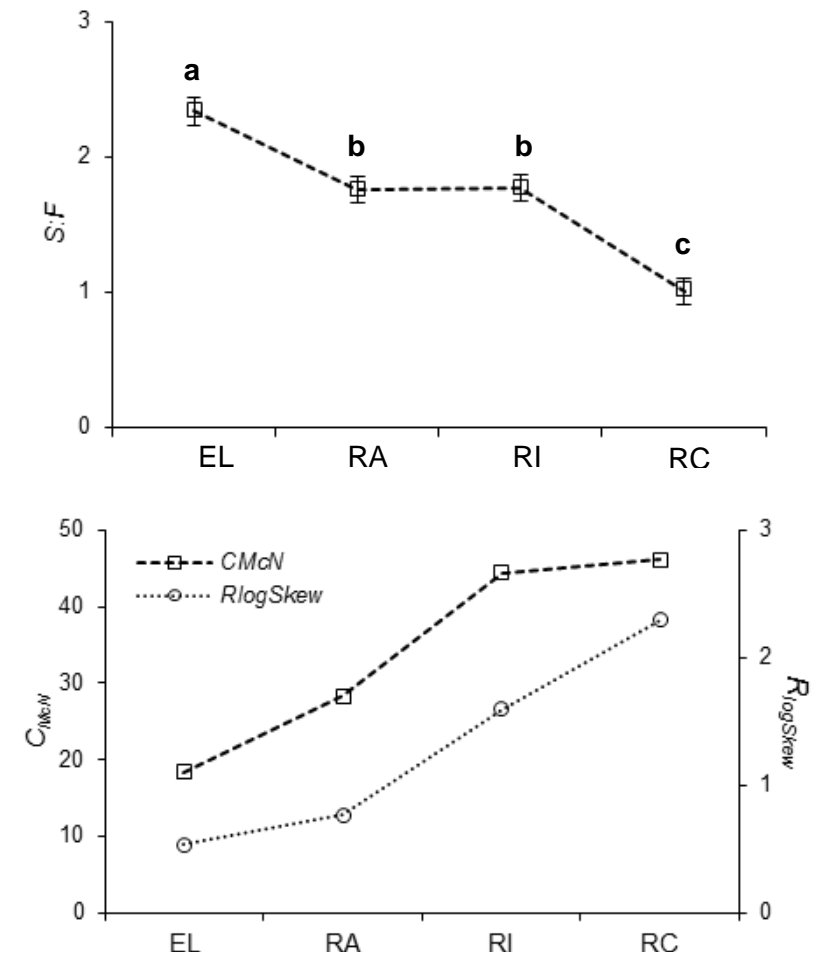

Las líneas verticales indican el error estándar de la media y las letras diferentes las diferencias significativas de las medias entre unidades de paisaje (prueba LSD, $p<0,05$ )

\section{Figura $N^{\circ} 6$}

RELACIÓN ESPECIES Y FAMILIAS ( $S: F$ ) (ARRIBA) Y DOMINANCIA DE ESPECIES ( $\left.C_{M C N}\right)$ Y PRESENCIA DE ESPECIES RARAS ( R $_{\text {LOGSKEW }}$ (ABAJO) EN LA ESTEPA-HERBÁCEO-ARBUSTIVA (EL) Y LOS RODALES ABIERTO (RA), INTERMEDIO (RI) Y CERRADO (RC)

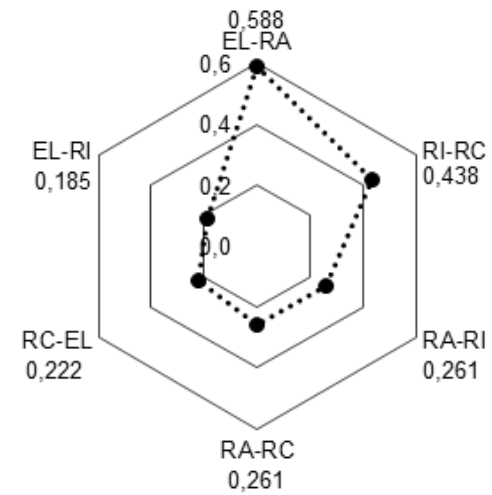

Figura $\mathrm{N}^{\circ} 7$

ÍNDICE DE SIMILITUD DE SøRENSEN EN LA ESTEPA-HERBÁCEO-ARBUSTIVA (EL) Y LOS RODALES ABIERTO (RA), INTERMEDIO (RI) Y CERRADO (RC) 


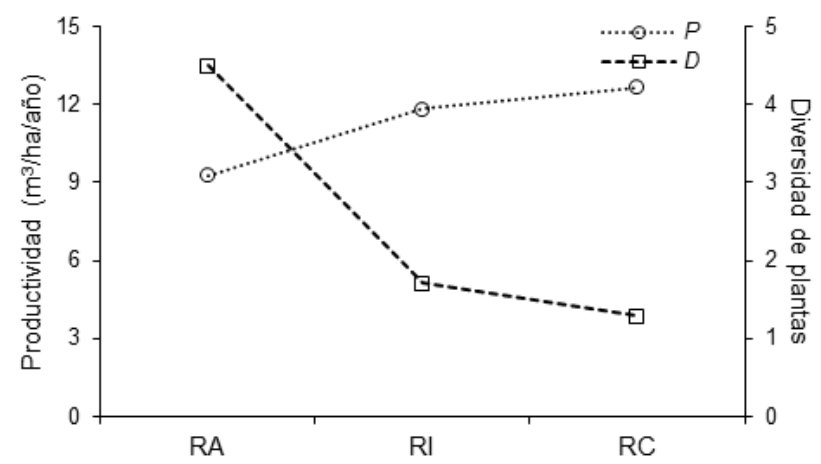

Figura $N^{\circ} 8$

PRODUCTIVIDAD DE MADERA (P) Y DIVERSIDAD DE PLANTAS (D)
EN LOS RODALES ABIERTO (RA), INTERMEDIO (RI) Y CERRADO (RC)

\section{DISCUSIÓN}

La baja radiación que alcanzó el suelo del rodal cerrado (2\%) fue probablemente debido a una alta reflexión y absorción de la luz causada por una alta cobertura del dosel (91\%) (Baldocchi et al., 1986, Canham et al., 1990). El dosel más desarrollado también implicaría mayores tasas de intercepción, evapotranspiración y consumo de agua (Rose, 1996; Le Maitre et al., 1999; Weigandt et al., 2015), que explicaría la reducción del contenido hídrico del suelo medido durante la estación seca $(9,6 \%)$.

Sin embargo, una estructura más densa del rodal también promovería una menor temperatura del aire dentro de este, reduciendo la evaporación y compensando parcialmente las pérdidas de agua a través de la canopia (Canham et al., 1990; Geiger et al., 2003).

La disminución de la cobertura de copas provocó un aumento de la luminosidad en el suelo del bosque, y probablemente afectó el contenido de agua del suelo para alcanzar niveles similares a los de la estepa. El rodal abierto presentó $29 \%$ de cobertura del dosel, el $42 \%$ de luz, y $20 \%$ de humedad del suelo, mientras que la humedad del suelo de la estepa fue $18 \%$. En el rodal cerrado, los valores más bajos de radiación y humedad del suelo habrían promovido la mayor cantidad de suelo desnudo (89\%), que disminuyó en el rodal abierto $(28 \%)$ hasta alcanzar un valor equivalente al de la estepa.

El rodal cerrado presentó valores muy bajos de riqueza $(3,6)$ y diversidad de especies de plantas $(1,3)$, cantidad de familias $(3,4)$ y relación especies y familias $(1,0)$, y valores altos de dominancia de especies $\left(C_{M c N}=46,1\right.$ y $\left.R_{\text {logskew }}=2,3\right)$ en comparación con los de la estepa $(14,8$; 6,$1 ; 7,6 ; 2,3 ; 18,4$ y 0,5 , respectivamente). La menor semejanza específica entre unidades de paisaje se produjo entre la estepa y el rodal intermedio $\left(C_{S}=0,19\right)$ y cerrado $\left(C_{S}=0,22\right)$. Estos valores mejoraron a medida que la cobertura aérea disminuyó en el rodal intermedio y abierto y aumentó la similitud entre la estepa y el rodal abierto $\left(C_{S}=0,59\right)$.

Estos fenómenos estarían relacionados con las posibilidades restringidas de establecimiento de las plantas en el rodal cerrado debido a la escasez de recursos aéreos y subterráneos esenciales como la luz y el agua. Aunque la alta frecuencia de especies introducidas en el rodal cerrado $(90 \%)$ se asoció a la especie exótica $P$. ponderosa, la cantidad de esta categoría de planta con relación al total fue muy bajo $(2 / 16)$ y comparable con el de la estepa (4/38). El rodal de pino no proporcionaría un hábitat particularmente ventajoso para las hierbas y arbustos introducidos, lo que reduce la posibilidad de invasión biológica. 


\section{CONCLUSIONES}

La menor producción final de madera del rodal abierto luego de un ciclo productivo de 36 años se asoció a la subocupación del sitio forestal, debido a la reducida densidad de árboles. El rendimiento aumentó a medida que el espacio de crecimiento fue ocupado por una mayor cantidad de individuos en el rodal intermedio y cerrado. Sin embargo, la mayor producción implicó un empobrecimiento de la diversidad biológica y otras variables asociadas, teniendo en cuenta la relación inversa que existió entre los indicadores de conservación ambiental y crecimiento a escala de rodal. Esta correlación negativa se debe tener en cuenta para implementar escenarios de sustentabilidad en estos hábitats antropogénicos.

El régimen silvicultural adecuado de manejo debería corresponder a una situación intermedia entre la del rodal abierto e intermedio. La técnica de la programación lineal podría encontrar el régimen de gestión sustentable que conduciría en forma simultánea a un rodal que mantenga valores adecuados de diversidad y productividad. La función objetivo debería maximizar el valor actual neto aplicando distintos escenarios de manejo y teniendo en cuenta restricciones asociadas al flujo de volumen y el mantenimiento de la diversidad (Buongiorno y Gilless, 2003; Rojas Ponce et al., 2007).

Las estrategias que promueven el mantenimiento de la diversidad, estructural y composicional de especies, formas de vida, grupos ecológicos y hábitats, a escala de rodal involucran la implementación de prácticas silviculturales de selección y asociadas a espaciamientos amplios de árboles, con raleos precomerciales intensivos y rotaciones extendidas, y de una zonificación de rodales con diferentes rotaciones (Quine y Humphrey, 2005; Lindenmayer et al., 2006; Brockerhoff et al., 2008).

La compatibilidad entre la conservación de una mayor variedad de especies silvestres y la producción de madera requiere esencialmente mantener rodales más abiertos a lo largo del ciclo de corta. Aunque tales proyectos presenten una productividad menor, tendrán valores ecosistémico y estéticos mayores que podrán aumentar la posibilidad de sostenerlos económicamente por parte de la sociedad.

\section{RECONOCIMIENTOS}

Este estudio fue financiado por la Universidad Nacional del Comahue y la Unidad de Cambio Rural (UCAR) del Ministerio de Agricultura, Ganadería y Pesca de la Nación. Agradecemos a A. Medina, L. Chauchard, R. González Musso y A. Suárez la colaboración, durante las diferentes instancias de la investigación, y a la Corporación Forestal Neuquina por permitir colectar datos en el área de estudio.

\section{REFERENCIAS}

AIC, 2012. Temperaturas medias mensuales y precipitaciones totales mensuales. Informe técnico. Autoridad Interjurisdiccional de las Cuencas de los ríos Limay, Neuquén y Negro. Cipolletti. 48 p.

Andenmatten, E. y Letourneau, F., 2003. Predicción y proyección del rendimiento de pino ponderosa en las provincias de Chubut y Río Negro, Argentina. Quebracho 10: 14-25.

Andenmatten, E.; Letourneau, F. y Getar, E., 2007. Simulador forestal para Pseudotsuga menziesii (Mirb) Franco y Pinus ponderosa (Laws) en Patagonia Argentina. En: H. Gonda, M. Davel, G. Loguercio y O. Picco (eds). I Reunión sobre forestación en la Patagonia, Esquel. p. 374-380.

Baldocchi, D.; Hutchison, B.; Matt, D. and McMillen, R., 1986. Seasonal variation in the statistics of photosynthetically active radiation penetration in an Oak--Hickory forest. Agricultural and Forest Meteorology 36: 343-361.

Bremer, L. and Farley, K., 2010. Does plantation forestry restore biodiversity or create green deserts? A 
synthesis of the effects of land-use transitions on plant species richness. Biodiversity Conservation 19: 3893-3915.

Brockerhoff, E.; Jactel, H.; Parrotta, J. A.; Quine, C. P. and Sayer, J., 2008. Plantation forests and biodiversity: Oxymoron or opportunity? Biodiversity Conservation 17: 925-951.

Buongiorno, J. and Gilless, J. K., 2003. Decision methods for forest resource management. Elsevier Science. New York. 439p.

Cabrera, A., 1971. Fitogeografía de la República Argentina. Boletín de la Sociedad Argentina de Botánica 14: 1 42.

Canham, C. D.; Denslow, J. S.; Platt, W. J.; Runkle, J. R.; Spies, T.A. and White, P. S., 1990. Light regimes beneath closed canopies and tree-fall gaps in temperate and tropical forests. Canadian Journal of Forest Research 20: 620-631.

CFI, 2009. Inventario del bosque implantado de la provincia de Neuquén. Consejo Federal de Inversiones, Ministerio de Desarrollo Territorial. Neuquén. $65 p$.

Corley, J.; Sackmann, P.; Rusch, V.; Bettinelli, J. and Paritsis, J., 2006. Effects of Pine silviculture on the ant assemblages (Hymenoptera: Formicidae) of the Patagonian steppe. Forest Ecology and Management 222: 162166.

Daniel, T. W.; Helms, J. A.; and Baker, F. S., 1979. Principles of silviculture. McGraw-Hill. New York. 521p.

Dezzotti, A.; Sbrancia, R.; Mortoro, A. y Monte, C., 2009. Invasión biológica de Pinus ponderosa y Pinus contorta: estudio de caso de una plantación en la Patagonia noroccidental. Investigación Agraria Serie Forestal 18(2): 181-189.

FAO, 2014. El estado de los bosques del mundo. Food and Agriculture Organization. Roma. 132p.

Feddema, J. J.; Oleson, K. W.; Bonan, G. B.; Mearns, L. O.; Buja, L. E.; Meehl, G. A. and Washington, W. M., 2005. The importance of land-cover change in simulating future climates. Science 310: 1674-1678.

Frugoni, M. C.; Dezzotti, A.; Medina, A.; Sbrancia, R. and Mortoro, A., 2016. Design and evaluation of an afforestation project based on geopedologic and ecological information in north-western Patagonia, Argentina. In: Zinck, A.; Metternicht, G.; Bocco, G. and del Valle, H. (eds), Geopedology: an integration of geomorphology and pedology for soil and landscape studies. Springer, Berlin, pp 480-504.

Geiger, R.; Aron, R. H. and Todhunter, P., 2003. The climate near the ground. Rowman and Littlefield Publishers. Lanham. 584p.

Gyenge, J. E.; Fernández, M. E.; Licata, J.; Weigandt, M.; Bond, B. J. y Schlichter, T., 2011. Uso del agua y productividad de los bosques nativos e implantados en el NO de la Patagonia: aproximaciones desde la ecohidrología y la ecofisiología. Ecología Austral 21: 271-284.

Hansen, M.; Stehman, S. and Potapov, P., 2010. Quantification of global gross forest cover loss. Proceedings of the National Academy of Sciences of the USA 107(19): 8650-8655.

IBD, 2015. Flora del Conosur. Instituto de Botánica Darwinion. Academia Nacional de Ciencias Exactas, Físicas y Naturales - CONICET. Disponible en http://www2.darwin.edu.ar. Acceso 30 Nov. 2015

Jost, L.; Chao, A. and Chazdon, R. L., 2010. Compositional similarity and $\beta$ (beta) diversity. En: Biological diversity: frontiers in measurement and asssessment. A. E. Magurran and B. J. McGill (eds.). Oxford University Press. Oxford. p. 66-84.

Kanowski, P. J., 1997. Afforestation and plantation forestry for the 21 st century. Proceedings $11^{\circ}$ Congreso Forestal Mundial. Antalya.

Kent, M., 2011. Vegetation description and data analysis: a practical approach. Wiley-Blackwell, New York. 428p.

La Manna, L.; Buduba, C.; Gigli, A. y Rostagno, C. M., 2013. Efecto de las plantaciones sobre la erosión hídrica potencial en suelos degradados de la Región Andino Patagónica. Actas II Congreso Internacional Agroforestal Patagónico. Calafate. p. 9.

Laclau, P., 2003. Biomass and carbon sequestration of Ponderosa Pine plantations and native Cypress forests in northwest Patagonia. Forest Ecology and Management 180: 317-333. 
Lantschner, M. V.; Rusch, V. and Peyrou, C., 2008. Bird assemblages in Pine plantations replacing native ecosystems in NW Patagonia. Biodiversity Conservation 17: 969-989.

Lantschner, M. V.; Rusch, V. and Hayes, J. P., 2011. Influences of Pine plantations on small mammal assemblages of the Patagonian forest-steppe ecotone. Mammalia 75: 249-255.

Le Maitre, D.; Scott, D. and Colvin, C., 1999. A review of information on interaction between vegetation and groundwater. Water 25(2): 137-152.

Lindenmayer, D.; Franklin, J. and Fischer, J., 2006. General management principles and a checklist of strategies to guide forest biodiversity conservation. Biological Conservation 131: 433-445.

Lindquist, E.; D’Annunzio, R.; Gerrand, A.; MacDicken, K.; Achard, F.; Beuchle, R.; Brink, A.; Eva, H.; Mayaux, P.; San-Miguel-Ayanz, J. and Stibig, H., 2012. Global forest land-use change 1990 - 2005. Forestry Paper 169. FAO. Roma. 53p.

Loguercio, G.; Gonda, H. y Jovanovski, A., 2011. Necesidades de manejo de los bosques plantados en las provincias de Neuquén, Rio Negro y Chubut. Revista Producción Forestal 1: 13-17.

Magurran, A. and McGill, B., 2011. Biological diversity: Frontiers in measurement and assessment. Oxford University Press. 368p.

MAGyP, 2014. Argentina: Plantaciones forestales y gestión sostenible. Unidad de Cambio Rural, Ministerio de Agricultura, Ganadería y Pesca de la Nación. Buenos Aires. 15p.

McGill, B., 2003. Does mother nature really prefer rare species or are log-left-skewed SAD's a sampling artefact? Ecology Letters 6: 766-773.

McNaughton, S. J. and Wolf, L. L., 1970. Dominance and the niche in ecological systems. Science 167: 131139.

Nosetto, M. D.; Jobbágy, E. G. and Paruelo, J. M., 2006. Carbon sequestration in semiarid rangelands: comparison of Pinus ponderosa plantations and grazing exclusion in NW Patagonia. Journal of Arid Environments 67: $142-156$.

Pan, Y.; Birdsey, R.; Fang, J.; Houghton, R.; Kauppi, P.; Kurz, W.; Phillips, O.; Shvidenko, A.; Lewis, S.; Canadell, J.; Ciais, P.; Jackson, R.; Pacala, S.; McGuire, D.; Piao, S.; Rautiainen, A.; Sitch, S. and Hayes, D., 2011. Large and persistent carbon sink in the world's forests. Science 333: 988-993.

Paritsis, J. and Aizen, M., 2008. Effects of exotic conifer plantations on the biodiversity of understory plants, epigeal Beetles and birds in Nothofagus dombeyi forests. Forest Ecology and Management 255: 1575-1583.

Peel, M.C.; Finlayson, B. L. and McMahon, T. A., 2007. Updated world map of the Köppen-Geiger climate classification. Hydrological Earth System Science 11: 1633-1644

Price, R.; Liston, A. and Strauss, S., 1998. Phylogeny and systematics of Pinus. En: Ecology and biogeography of Pinus. D. Richardson ed. Cambridge University Press. Cambridge. p. 49-68.

Quine, C. P. and Humphrey, J., 2010. Plantations of exotic tree species in Britain: Irrelevant for diversity of novel habitats for native species? Biodiversity Conservation 19: 1503-1512.

Rojas Ponce, Y.; García Robredo, F. y Ruiz-Tagle Molina, M., 2007. Un modelo de gestión para la conservación de la biodiversidad forestal en renovales de roble-raulí-coigüe. INFOR 13(3): 501-515.

Rose, C. L., 1996. Forest canopy - atmosphere interactions. Northwest Science 70: 7-14.

SAMLA, 1996. Sistema de apoyo metodológico para laboratorio de análisis de suelos y aguas. Dirección de Producción Agrícola. Secretaría de Agricultura, Ganadería, Pesca, Alimentos y Forestación. Buenos Aires. Disponible en http://www.minagri.gob.ar/site/agricultura/samla. Acceso 30 Nov 2015.

Sarasola, M.; Rusch, V.; Schlichter, T. y Ghersa, C., 2006. Invasión de coníferas forestales en áreas de estepa y bosques de la cordillera en la región Andino Patagónica. Ecología Austral 16: 143-156.

SAyDS, 2007. Primer inventario nacional de bosques nativos. Secretaría de Ambiente y Desarrollo Sustentable. Buenos Aires. 126p. 
Simpson, E. H., 1949. Measurement of diversity. Nature 163: 688.

Sørensen, T., 1948. A method of establishing groups of equal amplitude in plant sociology based on similarity of species content and its application to analyses of the vegetation on Danish commons. Biologiske Skrifter 5: 1-34.

Weigandt, M.; Gyenge, J.; Fernández, M. E.; Varela, S. and Schlichter, T., 2015. Afforestations and wetlands, are they a good combination? Study of water fluxes in two cases of Patagonian wetlands. Ecohydrology 8(3): 416425. 